\title{
Surveys and Analysis of RFI in The Smos Context
}

\section{Skou, Niels; Balling, Jan E.; Søbjærg, Sten Schmidl; Kristensen, Steen Savstrup}

\section{Published in:}

International Geoscience and Remote Sensing Symposium

Link to article, DOI:

10.1109/IGARSS.2010.5649838

Publication date:

2010

Document Version

Publisher's PDF, also known as Version of record

Link back to DTU Orbit

Citation (APA):

Skou, N., Balling, J. E., Søbjærg, S. S., \& Kristensen, S. S. (2010). Surveys and Analysis of RFI in The Smos Context. In International Geoscience and Remote Sensing Symposium (pp. 2011-2014). IEEE.

https://doi.org/10.1109/IGARSS.2010.5649838

\section{General rights}

Copyright and moral rights for the publications made accessible in the public portal are retained by the authors and/or other copyright owners and it is a condition of accessing publications that users recognise and abide by the legal requirements associated with these rights.

- Users may download and print one copy of any publication from the public portal for the purpose of private study or research.

- You may not further distribute the material or use it for any profit-making activity or commercial gain

- You may freely distribute the URL identifying the publication in the public portal 


\title{
SURVEYS AND ANALYSIS OF RFI IN THE SMOS CONTEXT.
}

\author{
Niels Skou, Jan E. Balling, Sten S. Søbjarg, and Steen S. Kristensen \\ Danish National Space Center, B 348. \\ Technical University of Denmark. \\ DK 2800 Lyngby, Denmark. \\ Phone: (45) 452537 68, Fax: (45) 45931634 \\ e-mail:ns@space.dtu.dk
}

\begin{abstract}
Several soil moisture and sea salinity campaigns, including airborne L-band radiometer measurements, have been carried out in preparation for the ESA Soil Moisture and Ocean Salinity (SMOS) mission. The radiometer used in this context is fully polarimetric and is capable of detecting Radio Frequency Interference (RFI) using the kurtosis method. Analyses have shown that the kurtosis method generally detects RFI in an efficient manner, even though it has its shortcomings. Hence, other detection methods have been investigated as well. In particular, inspection of the $3^{\text {rd }}$ and $4^{\text {th }}$ Stokes parameters shows promising results possibly as a complement to the kurtosis method. The kurtosis method, however, cannot be used with SMOS data. Since SMOS is fully polarimetric, the 3rd and 4th Stokes parameter method is an option, and this has been used on a recent, fully polarimetric SMOS data set. Finally, a discussion of the variable incidence angle signature algorithm, and the possibility of using this as RFI indicator, is carried out.
\end{abstract}

Index Terms- SMOS, L-band, microwave, radiometer, radio-frequency interference (RFI), kurtosis.

\section{INTRODUCTION}

In November 2009, the European Space Agency (ESA) launched its SMOS satellite mission whose purpose is to measure sea-surface salinity and soil moisture by means of L-band radiometry [1]. Due to many active services operating at L-band frequencies, the mission is highly susceptible to man-made radio-frequency (RFI). Hence, methods for the detection and mitigation of RFI are of prime interest.

Since radiometric data acquired from space at Lband have only recently become available upon the launch of SMOS, the analysis of different RFI detection and mitigation strategies discussed in this paper is mainly based on experience gained during the series of airborne campaigns, carried out in support of the SMOS mission during the period 2005-2008. These experiments and the first findings are described in [2], and the present paper can be seen as a continuation of that reference.

\section{EMIRAD-2 RADIOMETER SYSTEM}

The EMIRAD-2 polarimetric radiometer system [3] is developed and operated by the Technical University of Denmark. The specifications of the instrument are as follows:

Correlation radiometer with sub-harmonic sampling and fully polarimetric, i.e. all four Stokes parameters.

The bandwidth corresponds to the full $27 \mathrm{MHz}$ protected band, and the integration time is $1 \mathrm{msec}$.

Two antenna beams: one pointing nadir and one pointing aft with $40^{\circ}$ incidence angle.

The instrument was installed in a Short SC-7 Skyvan aircraft owned and operated by the Aalto University School of Science and Technology (former name: Helsinki University of Technology).

The radiometer system detects RFI using the kurtosis algorithm [4]. In an ideal situation, i.e. without any external man-made disturbances, a microwave radiometer detects natural thermal emissions i.e. stochastic processes, and the voltage measured by the radiometer has a Gaussian distribution. By contrast, man-made signals are assumed to have a non-Gaussian distribution. Thus, an RFIcontaminated signal received by the radiometer will have a distribution which deviates more or less from Gaussian.

The kurtosis algorithm makes use of higher-order central moments of the received radiometer signal. The kurtosis ratio $k$ is defined as the ratio of the $4^{\text {th }}$ order central moment to the squared $2^{\text {nd }}$ order central moment. The kurtosis ratio ideally equals 3 for a signal with a purely Gaussian distribution.

In practice, a finite number of samples are used for estimating the kurtosis ratio, which makes the ratio behave as a random variable. It will therefore have a standard deviation associated with it. This standard deviation is found when observing the internal calibration load. When observing the antenna, data points having a kurtosis deviating more than 4 standard deviations from 3 are flagged as RFI contaminated. 


\section{EXPERIENCES WITH KURTOSIS-BASED RFI DETECTION}

In order to illustrate the performance of the current kurtosisbased RFI detection scheme, an example has been provided in Figure 1. The data shown in the figure were acquired from the vertical channel of the nadir-looking antenna near Munich on April 18 $8^{\text {th }}, 2008$.

The data have been calibrated and plotted as brightness temperatures (TB) vs. UTC time. All samples which have been flagged as contaminated have been grouped together in the red top half of the plot, whereas the samples reported as being clean are shown in blue at the bottom of the plot.
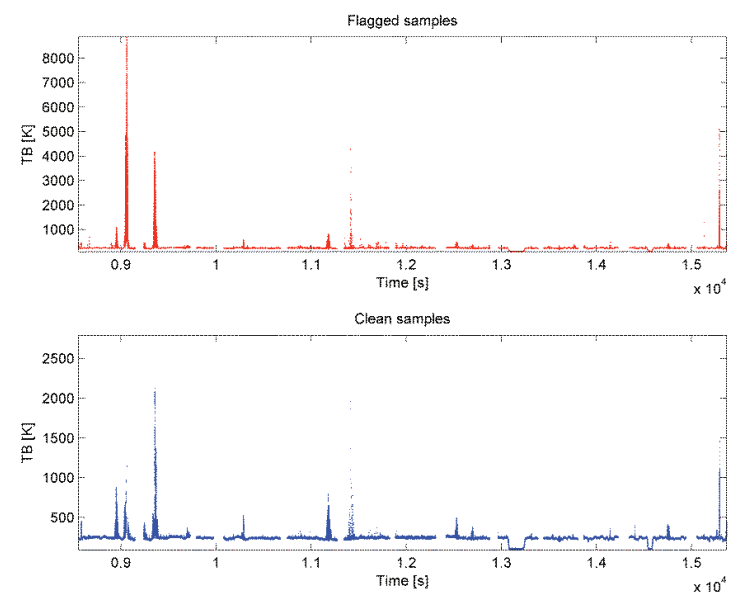

Figure 1: Data flagged as contaminated (top) and clean (bottom) by the current RFI detection scheme. 3.5\% of the samples in the data set have been flagged.

A large fraction of RFI contaminated samples have been flagged, but it is also evident that the so-called clean samples include many spikes that are obviously RFI not being flagged by the algorithm. Actually, in this example $0.6 \%$ of the "clean" samples are larger than $350 \mathrm{~K}$ - surely non-natural levels.

One easy way of improving the sensitivity of the algorithm is to lower the guard band around the kurtosis value 3 from 4 to f. ex. 0.5 standard deviations. This comes at a price since now $65 \%$ of the samples are flagged. Unfortunately still $0.3 \%$ of the clean samples are larger than $350 \mathrm{~K}$.

Another easy improvement is to flag not only samples having a kurtosis different from 3, but also neighboring samples. Flagging 30 neighbors leads to a similar disappointing result (21\% flagged, still 0.3\% "clean" samples larger than $350 \mathrm{~K}$ ).

The problem is probably that the kurtosis algorithm has some weaknesses: as an example, a pulsed sinusoidal signal with a low duty cycle will have a kurtosis ratio larger than three, whereas a continuous sinusoidal signal will give rise to a kurtosis ratio equal to 1.5 . If, however, a sinusoidal signal with a duty cycle of $50 \%$ is observed, it will have a kurtosis ratio of 3 , thus falsely indicating the presence of a signal with a Gaussian distribution, see Figure 2.

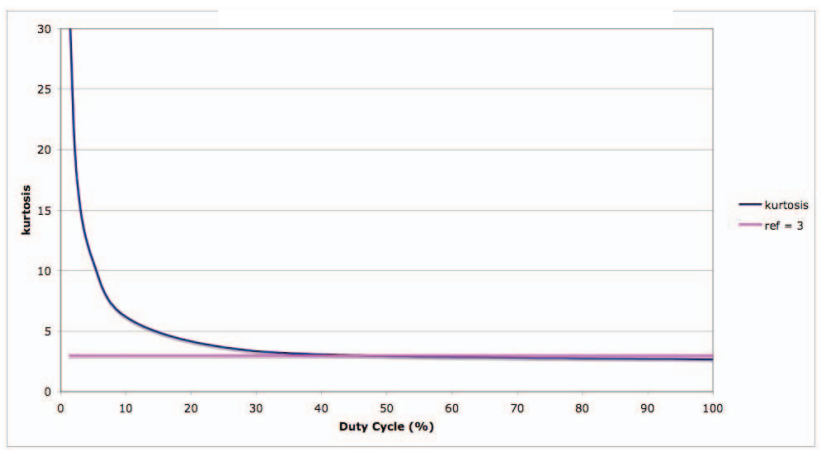

Figure 2: Blind spot, good sensitivity at low duty cycles, and poor sensitivity to large duty cycles

Note that $50 \%$ duty cycle means $50 \%$ within the time over which the radiometer calculates the kurtosis. This can be varied in the present system. Doing this, i.e. calculating kurtosis for different periods, and evaluating them together, should circumvent the blind spot problem. However, this is not the case using real data, and disappointing results, along the line as reported earlier in this chapter, is found. The problem is not the theoretical blind spot at $50 \%$ duty cycle, but rather the poor sensitivity at large duty cycles.

\section{INSPECTION OF STOKES PARAMETERS}

The $3^{\text {rd }}$ and $4^{\text {th }}$ Stokes parameters are expected to be very small at L-band for natural targets. During the analysis of EMIRAD-2 data it has been noticed that the $3^{\text {rd }}$ and $4^{\text {th }}$ Stokes parameters often grow to large values when RFI contamination is present. Figure 3 shows this phenomenon: For each sample in the data set (the same as used before), its deviation from the mean brightness temperature of the entire data set has been plotted as a function of its associated $3^{\text {rd }}$ and $4^{\text {th }}$ Stokes parameters. Also, each data sample has been color-coded in order to show its status upon application of the kurtosis-based RFI detection method. In order to avoid effects on the $3^{\text {rd }}$ Stokes parameter related to aircraft attitude, only data samples acquired at times with minimal roll and pitch have been used as the basis of the figure. Please note that the points are actually very concentrated near $(0,0)$ : $98 \%$ of the point have $3^{\text {rd }}$ and $4^{\text {th }}$ stokes values within $0 \pm 10 \mathrm{~K}$.

It is observed that a correspondence between elevated values of brightness temperatures and of the $3^{\text {rd }}$ and $4^{\text {th }}$ Stokes parameters certainly does exist - furthermore, many extreme cases of TB deviations are detected by the kurtosis-based RFI detection method. It is also noted, however, that a number of extreme TB deviations does not have an associated polarimetric value away from zero. 

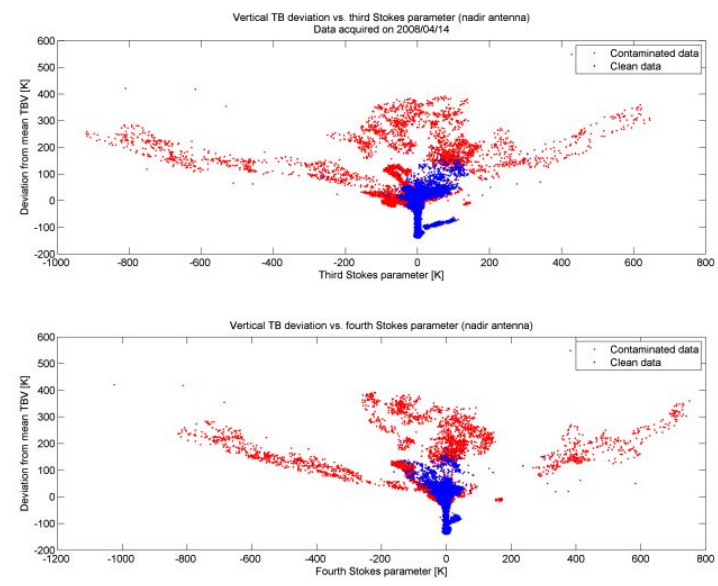

Figure 3: Vertical TB deviation vs. observed $3^{\text {rd }}$ (top) and $4^{\text {th }}$ (bottom) Stokes parameters. Blue dots are clean according to kurtosis, red dots flagged as RFI contaminated.

A suitable method for RFI detection is to look for data points having both the $3^{\text {rd }}$ and the $4^{\text {th }}$ Stokes parameters to be $0 \pm 10 \mathrm{~K}$, and regard them as clean. In order to provide a comparison of this Stokes parameter algorithm and the kurtosis-based algorithm, Figure 7 shows the same data set as was used before. It is clearly seen that visible peaks in the data set due to RFI are much more efficiently removed than when using the kurtosis-based method, and only $0.01 \%$ of the "clean" data are larger than $350 \mathrm{~K}$. Switching to this more efficient detection method, the percentage of data samples being flagged as contaminated increases from $3.5 \%$ to $15 \%$.

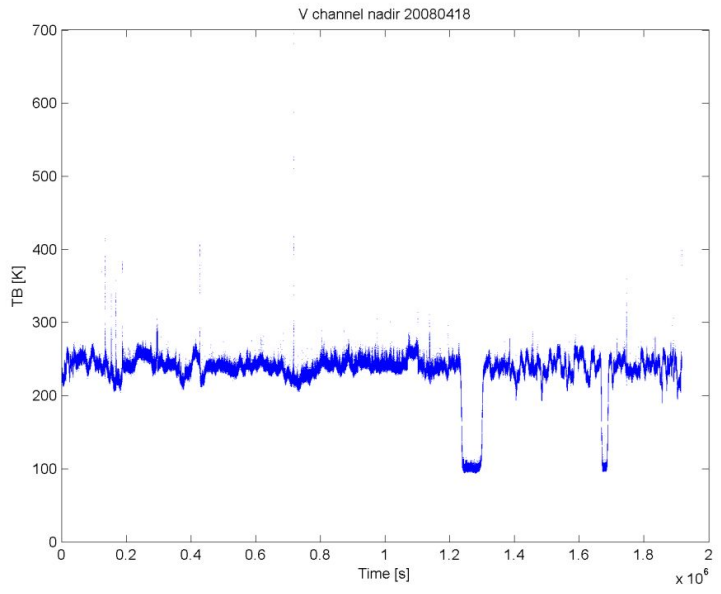

Figure 4: A data set cleaned by application of $3^{\text {rd }}$ and $4^{\text {th }}$ Stokes parameter thresholds of $\pm 10 \mathrm{~K}$. The percentage of samples flagged as contaminated amounts to $15 \%$. The percentage of obvious RFI candidates not being detected is less than $0.01 \%$

In addition to merely inspecting the values of the $3^{\text {rd }}$ and $4^{\text {th }}$ Stokes parameters, an additional approach to Stokes parameter inspection has been tested: When data samples are affected by RFI, the $3^{\text {rd }}$ and $4^{\text {th }}$ Stokes parameters exhibit not only elevated levels but also rapid fluctuations; in other words, RFI-contaminated segments have a higher standard deviation than clean ones. For the usual data set a "moving standard deviation" of its $3^{\text {rd }}$ and $4^{\text {th }}$ Stokes parameters have been calculated by means of a 149-sample wide sliding window. Then, suitable thresholds have been found: $\sigma\left(3^{\text {th }}\right.$ Stokes $)<5 \mathrm{~K}$ and $\sigma\left(4^{\text {th }}\right.$ Stokes $)<4 \mathrm{~K}$. Using this criterion results in a graph that looks a lot like Figure 4, with $14 \%$ of the data set being flagged as contaminated while only $0.001 \%$ of the "clean" data are larger than $350 \mathrm{~K}$.

\section{SMOS POLARIMETRIC DATA}

Figure 5 shows a SMOS snapshot covering Germany, Denmark and southern Sweden. All 4 stokes parameters are shown. Incidence angles vary from nadir out to some $60^{\circ}$ towards the upper edge of the snapshot.

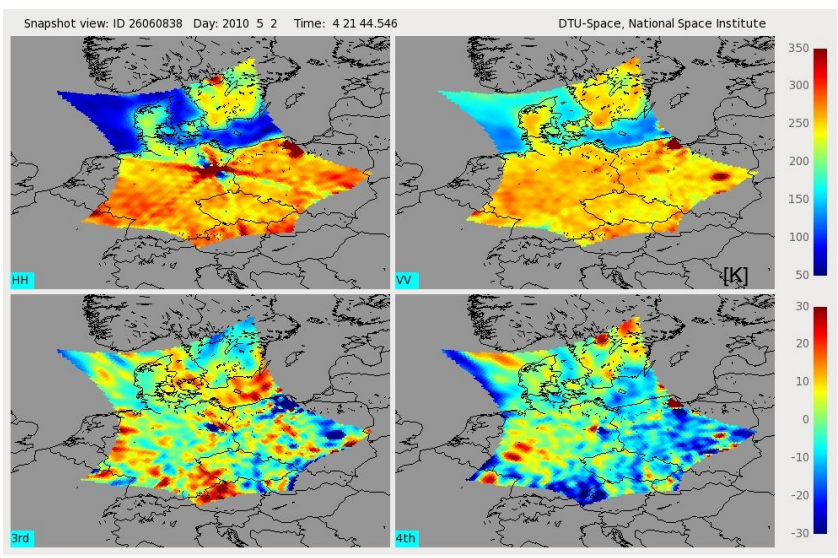

Figure 5: SMOS snapshot, fully polarimetric

A strong RFI source is seen near Berlin, and it spreads out over a large part of the snapshot - especially in the $\mathrm{H}$ pol. Image. Clear corresponding responses are seen in the $3^{\text {rd }}$ and $4^{\text {th }}$ Stokes channels. In order to compare the polarimetric behavior with that of the airborne situation, a scatter plot (a bit like the one shown in Figure 3) is generated and shown in Figure 6. Beware, the data set behind the plot is not the same as shown in Figure 5, but rather a similar scene taken from the so-called browse product. This product is brightness temperatures at $42.5^{\circ}$ incidence angle.

Figure 6 illustrates a behavior much like what was experienced with the airborne data. Bear in mind that the number of data points is very small in Figure 6 compared with Figure 3 due to much slower data rate of SMOS (1.2 sec. integration time). Anyway, the similarities are there to warrant a further investigation of the usefulness of polarimetric data for RFI flagging. Data sets from the SMOS Cal/Val campaign, carried out May - June 2010, are currently under investigation. 


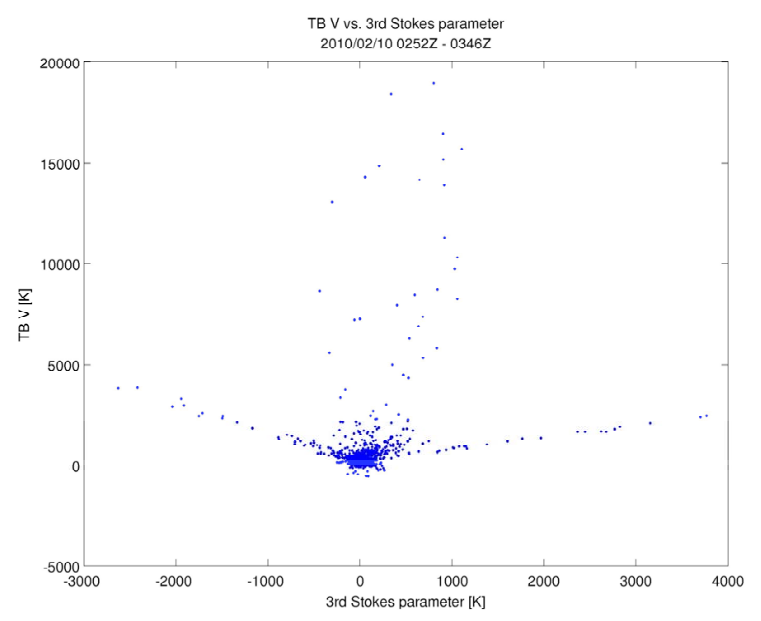

Figure 6: Vertically polarized brightness temperatures shown as a function of the $3^{\text {rd }}$ Stokes parameter.

\section{SMOS DATA, VARIBLE INCIDENCE ANGLE}

It can be argued that geophysical emission will have a certain incidence angle dependence, and RFI in general a very different one. Thus looking for unusual incidence angle behavior might constitute a possible RFI detection algorithm. As the snapshot moves over a certain point, f. ex. in central Denmark, the incidence angle behavior is revealed as shown in Figure 7.

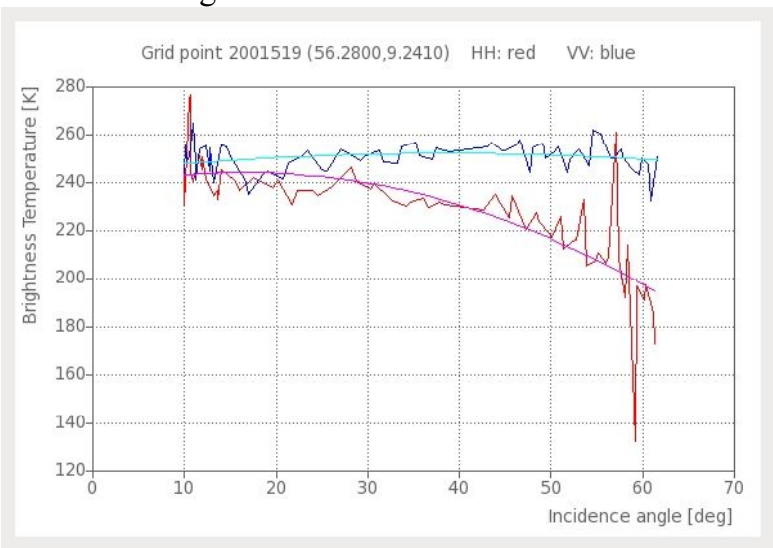

Figure 7: TB vs. incidence angle, central Denmark

$\mathrm{V}$ and $\mathrm{H}$ pol. are shown together with second order fits. RFI could be indicated near $10^{\circ}$ and in the $55-60^{\circ}$ range, and clean data seems present over most of the range. This corresponds well with the rather clean appearance of Denmark in Figure 5

Figure 8 shows a similar plot for Berlin. Severe RFI is indicated at several incidence angles, as could be expected from Figure 5, but even here there are parts of the range that might contain clean and useful data. This is currently under investigation, using the $\mathrm{Cal} / \mathrm{Val}$ data sets.

Before leaving this subject, it should be noted that actually the graphs in Figures 7 and 8 are also a function of time due to the way in which the snapshot sweeps across the ground. The combined effect of incidence angle and time complicates matters, and is subject for further analysis and discussions.

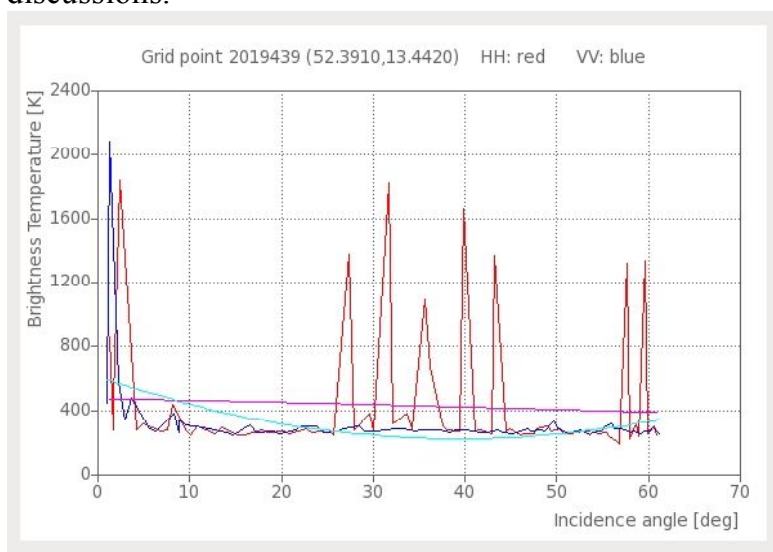

Figure 8: TB vs. incidence angle, Berlin

\section{CONCLUSIONS}

Different approaches to RFI detection have been tested on EMIRAD-2 data acquired during airborne campaigns.

It has been established that RFI detection based on kurtosis thresholds in general works well, but certain RFI spikes pass undetected. Several attempts at improving the algorithm by parameter tuning, have not been fruitful. RFI filtering based on inspection of the $3^{\text {rd }}$ and $4^{\text {th }}$ Stokes parameters has shown promising results, actually in some cases better that the kurtosis method. However, kurtosis has a unique ability to detect minor but still harmful RFI that go undetected by other algorithms, and a combination of kurtosis and polarimetry is suggested as a powerful detector.

Preliminary analysis of SMOS data indicate the usefulness of polarimetry also here, and additionally, the unusual incidence angle signature algorithm looks very promising.

\section{REFERENCES}

[1] "Special issue on the SMOS mission", IEEE Trans. Geosc. Remote Sens., vol. 46, no. 3, Mar. 2008

[2] N. Skou, S. Misra, J. E. Balling, S. S. Kristensen, and S. S. Søbjærg: "L-band RFI as Experienced During Airborne Campaigns in Preparation for SMOS", IEEE Trans. on Geosc. Remote Sens., Vol. 48, No. 3, pp. 1398-1407, Mar. 2010.

[3] J. Rotbøll, S. S. Søbjerg, and N. Skou: "A Novel L-band Polarimetric Radiometer Featuring Subharmonic Sampling" Radio Science, Vol 38, No. 3, May-June 2003, pp 11-1 - 11-7.

[4] C. S. Ruf, S. M. Gross, and S. Misra, "RFI detection and mitigation for microwave radiometry with an agile digital detector", IEEE Trans. Geosc. Remote Sens., vol. 44, no. 3, pp. 694-706, Mar. 2006. 